Um so wichtiger ist es, im Vorfeld zu prognostizieren, ob für den individuellen Patienten eine organerhaltende Therapieform geeignet ist. Im Einzelfall ist dies bislang nicht einfach. Die von Dietz geleitete deutsche LarynxorganerhaltStudiengruppe (DeLOS) entwickelte jetzt ein Bewertungsmodell, das die Entscheidungsfindung erleichtern soll. Der Score basiert auf Ergebnissen der DeLOS II-Studie [Wichmann G et al.: Eur J Cancer 2017; 72: 144-55]. Patienten mit lokal fortgeschrittenem Larynxkarzinom Stadium III oder IV erhielten zunächst nur einen Zyklus einer Induktions-Chemotherapie in Kombination mit bzw. ohne Cetuximab (Kurzinduktion). Patienten, bei denen sich endoskopisch eine
Schrumpfung der Tumoroberfläche von mindestens $30 \%$ zeigte, wurden als frühe Responder eingestuft und erhielten einen weiteren Zyklus der initialen Therapie sowie anschließend eine Radiotherapie. Bei den anderen wurde eine Salvagelaryngektomie durchgeführt. Das Volumen des Primärtumors sowie der Lymphknoten wurde mittels CT bestimmt, außerdem erfolgte eine PET.

$75 \%$ der Patienten waren frühe $\mathrm{Re}$ sponder. Es zeigte sich, dass eine frühe Laryngektomie bei fehlendem Ansprechen der Induktions-Chemotherapie sowie eine Tumorschrumpfung um mehr als $70 \%$ bei Respondern zu den besten Überlebensraten führten. Als Prädiktoren für einen langfristigen Larynxerhalt bei Res- pondern konnten verschiedene Parameter der endoskopischen, CT- und PET-Befunde eruiert werden. Aus diesen wurde ein Score entwickelt, der hilft, die Patienten mit den größten Chancen auf einen Larynxerhalt bzw. die Patienten zu identifizieren, die für ein larynxerhaltendes Therapieregime nicht geeignet sind. Eine solche Response-Evaluation nach Induktions-Chemotherapie könnte künftig therapeutisch wegweisend werden, betonte Studienleiter Dietz. Eine interdisziplinäre Zusammenarbeit sei dabei unerlässlich, um eine individualisierte, personalisierte Therapieentscheidung treffen zu können.

Dietz A.: Neue Strategien in der Therapie von Kopf-Hals-Tumoren

\section{Nachsorge: wesentlicher Bestandteil der onkologischen Versorgung}

D ie onkologische Nachsorge hat zum Ziel, ein Therapieversagen und Zweittumore zu identifizieren, aber auch das Management von Nebenwirkungen und die psychosoziale Situation zu verbessern.

Auch wenn keine Studien möglich sind, die direkte Effekte auf das Überleben von Tumorpatienten statistisch erfassen: Der allgemeine Benefit einer strukturierten Nachsorge sei unstrittig, betonte Prof. Dr. Claus Wittekindt, Hals-Nasen- und Ohrenklinik, Universitätsklinikum Gießen. Für die Nachsorge wurden verschiedene Standards erarbeitet, die jedoch wenig evidenzbasiert sind [Simo R et al.: J Laryngol Otol. 2016 May; 130(S2): S208-S211].

Bei Kopf-Hals-Tumoren ist eine Nachsorge über fünf Jahre Standard. Danach ist ein Therapieversagen sehr selten. Eine längere Nachsorge kann allerdings aufgrund besonderer Risiken und der Biologie der Erkrankung sinnvoll sein, beispielsweise bei HPV-positiven Tumoren oder beim adenoid-zystischen Karzinom. Auch auf Wunsch des Patienten wird nicht selten eine längere Nachsorge praktiziert.

Die Nachsorgeintervalle betragen in der Regel im ersten Jahr ein bis drei Monate und verlängern sich in den Fol- gejahren auf vier bis sechs Monate im vierten und fünften Jahr. Für verkürzte Nachsorgeintervalle sei in der Regel keine Reduktion von Morbidität oder Mortalität gesichert, erläuterte Wittekindt.

Fester Bestandteil der im Rahmen der Nachsorge durchgeführten Untersuchungen ist die Erhebung des Spiegelund Endoskopiebefundes. In Deutschland ist zudem eine Sonografie etabliert. Eine Schichtbildgebung wird meistens nur bei Beschwerden empfohlen. Sie erfolgt außerdem bei besonderem Bedarf, beispielsweise bei Tumorlokalisationen im Hypopharynx oder Nasopharynx, aber auch bei erhöhtem Risiko aufgrund der Biologie des Tumors oder Risikofaktoren wie starkem Rauchen. Viel diskutiert wird der Einsatz der PET-CT. Studiendaten zeigen, dass diese bei persistierenden pathologischen Halslymphknoten nach Radiochemotherapie vor einer Neck-Dissection sinnvoll ist. Ein Verzicht auf eine Salvage-Neck-Dissection bei negativem FDG-Uptake beeinträchtigt das Gesamtüberleben nicht [Mehanna H et al.: N Engl J Med 2016; 374 (15): 1444-54].

Für jährliche Röntgen-Thorax-Untersuchungen gäbe es keine Evidenz, so Wittekindt.
Zur Nachsorge zählt auch die standardisierte Erfassung von Nebenwirkungen und Toxizität der Tumortherapie, um entsprechende Maßnahmen in die Wege leiten zu können. Der Patient sollte außerdem über Maßnahmen der Rehabilitation beraten werden.

Eine besondere Bedeutung hat auch die Aufklärung über tumorspezifische Symptome, auf die der Patient achten sollte, da ein Therapieversagen in der Regel nicht durch einen symptomlosen pathologischen Befund allein auffällig wird.

Eine Untersuchung von Tumormarkern im Blut sei in der Nachsorge noch nicht etabliert, so Wittekindt. Ausnahme sind Schilddrüsenparameter bei Schilddrüsenkarzinomen und Untersuchungen auf EBV bei Nasopharynxkarzinomen.

Für jeden Patienten sollte ein strukturierter risikoadaptierter und individualisierter Nachsorgeplan erstellt werden, der durch ein multidisziplinäres Team in Kooperation mit Facharzt und Hausarzt umgesetzt werden sollte, fasste Wittekindt zusammen..

Wittekindt C.: Medizinischer Standard bei der Nachsorge maligner Tumoren im Kopf-Hals-Bereich in der Praxis

Berichterstattung: Angelika Bauer-Delto 